\title{
AD HOC CONSULTANTS - 2018
}

Adriana del Pilar Urbina Bonilla - Universidad Del Rosario, Bogotá-Colômbia

Adriana Benevides Soares - Universidade do Estado do Rio de Janeiro, Rio de Janeiro-RJ

Adriano Lemos Alves Peixoto - Universidade Federal da Bahia, Salvador-BA

Alana Augusta Concesso de Andrade - Universidade Federal de Minas Gerais, Belo Horizonte-BH

Alessandra Pimentel - Instituto Federal de Educação, Ciência e Tecnologia do Paraná, Palmas-PR

Alex Bacadini França - Universidade Federal de São Carlos, São Carlos-SP

Alex Sandro Gomes Pessoa - Universidade Federal de São Carlos, São Carlos-SP

Alexandre José de Souza Peres - Universidade Federal de Mato Grosso do Sul, Paranaíba-MS

Amália Raquel Pérez-Nebra - Centro Universitário de Brasília, Brasília-DF

Amanda Franco - Universidade de Aveiro, Aveiro-Portugal

Ana Cristina Resende - Universidade Federal de São Paulo, São Paulo-SP

Ana Maria Costa e Silva - Universidade do Minho, Minho-Portugal

Ana Carolina Braz - Universidade Federal de São Carlos, São Carlos-SP

Andréa Regina Rosin Pinola - Universidade Federal de São Carlos, São Carlos-SP

Andreia Schmidt - Universidade de São Paulo, Ribeirão Preto-SP

Andressa Melinda Becker da Silva - Universidade de Sorocaba, Sorocaba-SP

Angélica Miki Stein - Universidade Tecnológica Federal do Paraná, Curitiba-PR

Antonio Paulo Angelico - Universidade Federal de São João del-Rei, São João del-Rei-MG

Camila Negreiros Comodo - Instituto de Terapia por Contingências de Reforçamento, Campinas-SP

Carina Nunes Bossardi - Universidade do Vale do Itajaí, Itajaí-SC

Carla Sabrina Xavier Antloga - Universidade de Brasília, Brasília-DF

Carlos Daniel Barros - Universidade de Lisboa, Lisboa-Portugal

Carlos Henrique Sancineto da Silva Nunes - Universidade Federal de Santa Catarina, Florianópolis-SC

Carolina Villa Nova Aguiar - Escola Bahiana de Medicina e Saúde Pública, Salvador-BA

Carolina Severino Lopes da Costa - Universidade Federal de São Carlos, São Carlos-SP

Cesar Alexis Galera - Universidade de São Paulo, Ribeirão Preto-SP

Cícero Roberto Pereira - Universidade Federal da Paraíba, João Pessoa-PB 
Cintia Bragheto Ferreira - Universidade Federal do Triângulo Mineiro, Uberaba-MG

Clarissa Mendonça Corradi-Webster - Universidade de São Paulo, Ribeirão Preto-SP

Clarissa Pinto Pizarro Freitas - Universidade Salgado de Oliveira, Niterói-RJ

Clarisse Pereira Mosmann - Universidade do Vale do Rio dos Sinos, São Leopoldo-RS

Conceição Aparecida Serralha - Universidade Federal do Triângulo Mineiro, Uberaba-MG

Cristina Satiê de Oliveira Pátaro - Universidade Estadual do Paraná, Campo Mourão-PR

Cynthia Cassoni - Universidade do Estado do Rio de Janeiro, Rio de Janeiro-RJ

Daniel Bartholomeu - Centro Universitário FIEO, Osasco-SP

Daniela Margarida Fonseca Freitas - Universidade do Porto, Porto-Portugal

Deise Maria Leal Fernandes Mendes - Universidade do Estado do Rio de Janeiro, Rio de Janeiro-RJ

Denise Falcke - Universidade do Vale do Rio dos Sinos, São Leopoldo-RS

Denise Ruschel Bandeira - Universidade Federal do Rio Grande do Sul, Porto Alegre-RS

Diego Castrillon - Asociación Colombiana de Terapia Cognitiva ACOTEC - Medellín-Colômbia

Diogo Lamela - Universidade Lusófona do Porto, Porto-Portugal

Edna Lúcia Tinoco Ponciano - Universidade do Estado do Rio de Janeiro, Rio de Janeiro-RJ

Edna Maria Marturano - Universidade de São Paulo, Ribeirão Preto-SP

Fabiana Cia - Universidade Federal de São Carlos, São Carlos-SP

Fabiana Queiroga - Centro Universitário de Brasilia, Brasília-DF

Felipe Valentini - Universidade São Francisco, Campinas-SP

Fernanda Aguiar Pizeta - Universidade de São Paulo, Ribeirão Preto-SP

Fernanda Kimie Tavares Mishima-Gomes - Universidade de São Paulo, Ribeirão Preto-SP

Flávia de Lima Osório - Universidade de São Paulo, Ribeirão Preto-SP

Francisco Antonio Pereira Fialho - Universidade Federal de Santa Catarina, Florianópolis-SC

Gimol Benzaquen Perosa - Universidade Estadual Paulista Julio de Mesquita Filho, Botucatu-SP

Girlene Ribeiro de Jesus - Universidade de Brasília, Brasília-DF

Gleny Terezinha Duro Guimarães - Pontifícia Universidade Católica do Rio Grande do Sul, Porto Alegre-RS

Goiara Mendonça de Castilho - Universidade de Brasília, Brasília-DF

Guilherme Wendt - University of London, London- United Kingdom

Gustavo Levandoski - Universidade Federal da Grande Dourados, Dourados-MS 
Helga Loos-Sant'Ana - Universidade Federal do Paraná, Curitiba-PR

Heloísa Gonçalves Ferreira - Universidade Federal do Triângulo Mineiro, Uberaba-MG

Hudson Fernandes Golino - Universidade Salgado Filho, Niterói-RJ

Inês Maria Guimarães Nascimento - Universidade do Porto, Porto-Portugal

Irene Kazumi Miura - Universidade de São Paulo, Ribeirão Preto-SP

Ivana Casali - Universidade Federal de São Carlos, São Carlos-SP

Jeanny Joana Rodrigues Alves de Santana - Universidade Federal de Uberlândia, Uberlândia-MG

Jefferson Rodrigues Pereira - Centro Universitário Unihorizontes, Belo Horizonte-MG

Jesselyn Tashima - Universidade de Brasília, Brasília-DF

Joaquim Carlos Rossini - Universidade Federal de Uberlândia, Uberlândia-MG

José Egídio Barbosa Oliveira - Universidade de São Paulo, Ribeirão Preto-SP

José Egídio Oliveira - Escola Superior de Saúde do Alcoitão, Alcabideche-Portugal

Karen Cristina Alves Lamas - Universidade Salgado de Oliveira, Juiz de Fora-MG

Katya Luciane Oliveira - Universidade Estadual de Londrina, Londrina-PR

Larissa Horta Esper - Universidade de São Paulo, Ribeirão Preto-SP

Lícia Barcelos de Souza - Universidade de São Paulo, Ribeirão Preto-SP

Lina María Perilla-Rodríguez - Universidad Nacional de Colômbia, Bogotá-Colômbia

Lucas Cordeiro Freitas - Universidade Federal de São João del-Rei, São João del-Rei-MG

Lucas Dannilo Aragão Guimarães - Universidade Estadual do Piauí, Teresina-Piauí

Luciana Carla dos Santos Elias - Universidade de São Paulo, Ribeirão Preto-SP

Luciana Leonetti Correia - Universidade Federal da Grande Dourados, Dourados-MS

Luciene Alves Miguez Naiff - Universidade Federal Rural do Rio de Janeiro, Rio de Janeiro-RJ

Luciana Bezerra de Souza Gianasi - Universidade Federal do Sul e Sudeste do Pará, Marabá-PA

Marcela Mansur Alves - Universidade Federal de Minas Gerais, Belo Horizonte-MG

Márcia Maria Peruzzi Elia da Mota - Universidade do Estado do Rio de Janeiro, Rio de Janeiro-RJ

Maria Cristina Quintas Antunes - Universidade de Trás-os-Montes e Alto Douro, Vila Real-Portugal

Maria de Lima Salum e Morais - Instituto de Saúde, Núcleo de Práticas de Saúde, São Paulo-SP

Maria Cristina Triguero Velozo Teixeira - Universidade Presbiteriana Mackenzie, São Paulo-SP

Maria Cristina Ferreira - Universidade Salgado de Oliveira, Niterói-RJ 
Mariana Hasse - Universidade Federal de Uberlândia, Uberlândia-MG

Mariana Gonçalves Boeckel - Universidade Federal de Ciências da Saúde de Porto Alegre, Porto Alegre-RS

Marina Rezende Bazon - Universidade de São Paulo, Ribeirão Preto-SP

Marinéia Resende - Universidade Federal de Uberlândia, Uberlândia-MG

Marisa Matias - Universidade do Porto, Porto-Portugal

Marlene de Cássia Trivellato Ferreira - Centro Universitário Barão de Mauá, Ribeirão Preto-SP

Marta Regina Gonçalves Correia Zanini - Centro Universitário das Faculdades Associadas de Ensino, São João

da Boa Vista - SP

Nassim Chamel Elias - Universidade Federal de São Carlos, São Carlos-SP

Nelson Torro Alves - Universidade Federal da Paraíba, João Pessoa-PB

Olga Maria Piazentin Rolim Rodrigues - Universidade Estadual Paulista Júlio de Mesquita Filho, Bauru-SP

Paloma Pegolo de Albuquerque - Universidade Federal do Triângulo Mineiro, Uberaba-MG

Paola Passareli Carrazzoni - Centro Universitário Barão de Mauá, Ribeirão Preto-SP

Patricia Lorena Quiterio - Universidade do Estado do Rio de Janeiro, Rio de Janeiro-RJ

Patricia Leila dos Santos - Universidade de São Paulo, Ribeirão Preto-SP

Paula Lemes - Universidade Federal de Uberlândia, Uberlândia-MG

Rafael De Tilio - Universidade Federal do Triângulo Mineiro, Uberada-MG

Renata Silva de Carvalho Chinelato - Universidade Federal de Santa Catarina, Florianópolis-SC

Richardson Augusto Rosendo da Silva - Universidade Federal do Rio Grande do Norte, Natal-RN

Rodolfo Augusto Matteo Ambiel - Universidade São Francisco, Campinas-SP

Rosa de Jesus Ferreira Novo - Universidade de Lisboa, Lisboa-Portugal

Sara Cadavid Espinha - Universidad Del Rosario, Bogotá-Colômbia

Silvana Alba Scortegagna - Universidade de Passo Fundo, Passo Fundo-RS

Simone dos Santos Paludo - Universidade Federal do Rio Grande, Rio Grande-RS

Sônia Maria Guedes Gondim - Universidade Federal da Bahia, Salvador-BA

Susana Ines Nunez Rodriguez - Universidade Salgado de Oliveira, Niterói-RJ

Susana Maria Gonçalves Coimbra - Universidade do Porto, Porto-Portugal

Sylvia Domingos Barrera - Universidade de São Paulo, Ribeirão Preto-SP

Talita Pereira Dias - Centro Universitário de Votuporanga, Votuporanga-SP 
Tânia Abreu da Silva Victor - Universidade do Estado do Rio de Janeiro, Rio de Janeiro-RJ

Teresa Coutinho - Universidade Federal do Estado do Rio de Janeiro, Rio de Janeiro-RJ

Teresa Leal - Universidade do Porto, Porto-Portugal

Thelma Veloso - Universidade Estadual da Paraíba, Campina Grande-PB

Tiago Ferreira - Universidade do Porto, Porto-Portugal

Vicky Nogueira Pileggi - Universidade de São Paulo, Ribeirão Preto-SP 